\title{
Effectual Resource Allocation using Auction Mechanism in Cloud Computing
}

\author{
R. Ananthakumar, K. Kartheeban
}

\begin{abstract}
Cloud computing $(C C)$ is one of the fastest emerging technology. As we all know, cloud is a wide pool of resource which provides resources based on the users request. Any service can be provided as a service through cloud. As the cloud contains many resources, there may also wastage of resources. To reduce this wastage, cloud providers enter into auctioning of resources when the demand is high. Cloud computing includes distinct resources. Because of the complementary and supplementary effects between distinct assets, bidders have preferences not for just a single resource but also for a set of resources. Auctioning for a bundle of resources is called mergeable auction (MA). Dynamic resource allocation in on demand for a bundle of resource is proposed by using MA - PROVISION algorithm and the scenarios are simulated using Cloudsim, a simulator meant for cloud computing analysis.
\end{abstract}

Keywords-Cloud Computing, Auction, Bid, Dynamic Resource Allocation, Mergeable sale and Cloudsim.

\section{INTRODUCTION}

Distributed computing (DC) is the quickest rising worldview of disseminated processing. It consists of a figuring model and not innovation. It fully covered a web related figuring also clients need to compensate according to the usage. DC have numerous focal points similar to denial foundation speculation, stockpiling ability, versatility, reinforcement and recuperation capacities, whenever access with web, financially savvy, and so forth., Cloud gives numerous kinds of administrations that goes under the significant classes like framework, programming and stage.

As distributed computing diminishes the establishment costs, a significant number of the clients are changing over to distributed computing resource s. Presently multi day's anything i.e. information, stockpiling, observing, organize and so forth., can be given as an administration by means of DC acted as AaaS (Anything-as-a-Service) [3].

DC is a virtualized domain also gives benefits as Virtual mechanism (VM) examples. This VM objects are unmistakable. Cloud clients could pick this arrangement otherwise measure of examples it need. There are numerous resource s accessible, there might be a few Circumstances which prompt underutilization of these resources.Thus, an appropriate resource assignment method must complete to build the income of cloud supplier.

Revised Manuscript Received on December 16, 2019

R.Ananthakumar, Asst Prof., Department of CSE, Kalasalingam Institute of Technology, Krishnankoil - 626126,Tamilnadu, India, Email: r.ananth05@gmail.com

Dr.K.Kartheeban, Associate Prof., Department of CSE, Kalasalingam Academic Research Education, Krishnankoil 626126,Tamilnadu, India, Email: k.kartheeban@klu.ac.in

\section{ISSUES DURING RESORCE ALLOTMENT}

DC is chiefly prevalent in favour of adaptability also it can be achieved from end to end versatility also accessibility of administrations at whatever point within necessitate. It means to provide dependable, altered also great QoS factors in the progressively altering registering situations. To accomplish these objectives, resource designation is particularly critical. Legitimate resource designation picks up consumer loyalty moreover.

\section{A. Resource allotment Noteworthiness}

Resource allotment in $\mathrm{CC}$ is the way toward appointing $\mathrm{CC}$ resources to the asked for $\mathrm{CC}$ clients in excess of the web. Resource designation issues according to [13]:

a) Contention of Resource: additional clients endeavour to get to a similar resource in this way expanding rush hour gridlock.

b) Shortage of Resource: An adequate amount of resources are not availed.

c) Discontinuity of Resource: Prompts separation of resources towards a few clients along with they couldn't be dispensed near the people within necessitate.

d) Provisioning of overflow: Supplying a greater number of resources additional to the necessitate.

e) Provisioning of Underflow: Supplying fewer resources than asked for sum.

For defeat this kind of concerns in resource allotment DC, sell off system is utilized.

B. Allotment of Resource with sale

Sale is a procedure of purchasing in addition to offering merchandise via assembling offers in favour of the accessible resources. The most astounding proffer is given resource. Sale becomes possibly the most important factor while interest is higher also resources are fewer. Combination of purchaser as well as vender had allowed giving their assessment to resource they are during necessitate also with repetitive exchanges, an understanding is completed in favour of the instalment of resource. As in DC, the extensive groups of sources are no more utilized via clients in the direction of its complete usage. Likewise here scheduled interest circumstance, below usage of sources can occur on the off chance that we pursue FCFS (First Come First Serve) premise.

In this way, on the off chance that we utilize sell off instrument for resource designation, usage of resources and in addition suppliers has monetary advantages and legitimate assignment decreases wastage of resources [12].

Sale condition incorporates salesperson, representative, cloud supplier and client. Barker might be the dealer some of the time. It is portrayed during Figure. 1 underneath [1]. 


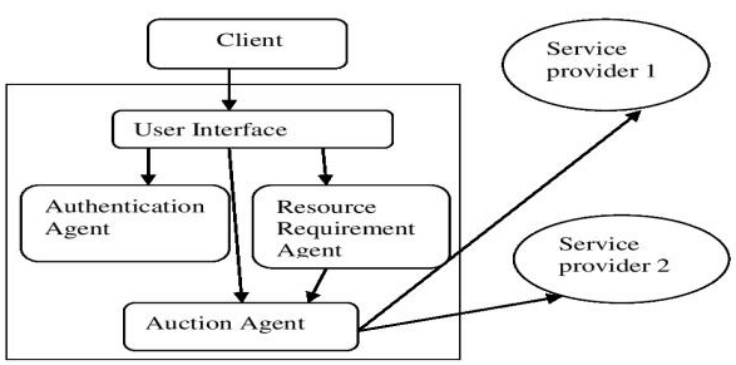

Figure 1: Sample sale methodology within cloud

Numerous remaining procedures in resource utilize inactive assignment with evaluating. The issues in inactive allotment included:

I. Deals with resource designation dependably support sellers. It might be a few circumstances while cases of clients with merchants are bungled.

ii. This is extremely hard in the direction of keep up SLA within settled valuing.

This issue could be overwhelmed through powerful resource allotment. Effectual source allotment gives monetary advantage to supplier also customer needs to compensate just in favour of utilization of sources. Honesty could be accomplished. This resource champ assurances are significant problem like this is NP - difficult issue.

From end to end virtualization, CC supplier could be capable design VMs dependent on client's inclinations. Several mixes of VM's could likewise survive acquired among accessible sources through supplier. Rather than solitary resource sale in favour of this heap of sources otherwise mixes is entitled mergeable resources [7].

Association of this document is specified via: In Part II, issues in allotment of resources are talked about. Segment III manages associated mechanism. Segment IV clarifies mergeable sale algorithm also Part $\mathrm{V}$ gives experimental outcomes and Part VI upcoming improvements of this effort is talked about.

\section{RELATED WORKS}

VM supplying via sales are tested through numerous scientists. Effectual supplying of resources is finished via Quiroz et al. during distributed web based bunching methods inside grid conditions. It enables clients in the direction of pick their endemic blends of sources. Progressively shifting condition is basis issue. Our projected framework manages present requests since clients [4].

Mergeable sale supported procedure in favour of source allotment inside grid are very much clarified via Daniel Grosu also Anubhav Das [5].Their effort clarifies as regards champ assurance issue throughout estimate methods in favour of auction. It includes grouping of greedy methodology in favour of assignment of sources which supports just to CC supplier's benefit. Daniel Lehmann et al.[6] planned greedy strategy in favour of champ assurance on behalf of determined sales. Proffers were organized dependent on standards during shipping request also no dual proffers had a similar standard. Deadlock circumstances are additionally analysed afterwards every distribution.

Honest of the method is clarified via AhuvaMualem et al.[2]. It gives with the aim of, at whatever point estimation of resource expands, utilization cost in favour of that source likewise increments. These arrangements among effectual valuing instrument of sources. Daniel Grosu also[ SharrukhZaman [9] proposed MA - LP in addition to MA GREEDY systems of mergeable sale in the direction of discover victor also in favour of effectual allotment.

This algorithm utilizes settled estimating instruments while it pursues inactive portion of sources. MA - LP component includes tackling of sale by direct programming. MA - GREEDY method utilizes is expanded edition of greedy allotment while this supports just suppliers. Late mechanisms of them [10] uncover MA - PROVISION method is the thickness supported unique source allotment.

Our planned effort takes out spurious client idea along with turns around value techniques in MA - PROVISION method. Experiments are finished via utilizing Cloud sim tool.

\section{MERGEABLE SALE SUPPORTED EFFECTUAL RESORCE ALLOTMENT}

Mergeable sale enables clients in the direction of offer in favour of a stack of sources otherwise bundles. Tendering sum is worried in favour of entire stack. Now and again, cloud supplier itself gives some default bundles like in Amazon [3] and clients can likewise have the inclinations for the bundles of their blends.

An example mergeable resource system is delineated in Fig. 2 [5]. Enabling the clients to express their inclinations expands the supplier's negation and income. Mergeable resource component is predominantly implied for enhancing monetary effectiveness and framework execution [11].

Our Projected effort, while source conflict otherwise opposition in support of resource builds, CC supervisor would require sale of sources between clients.

Clients get warning along with afterward begin to forward offers in support of sources. This mergeable resource supported component entitled MA - PROVISION gives data concerning portion of accessible resources to 


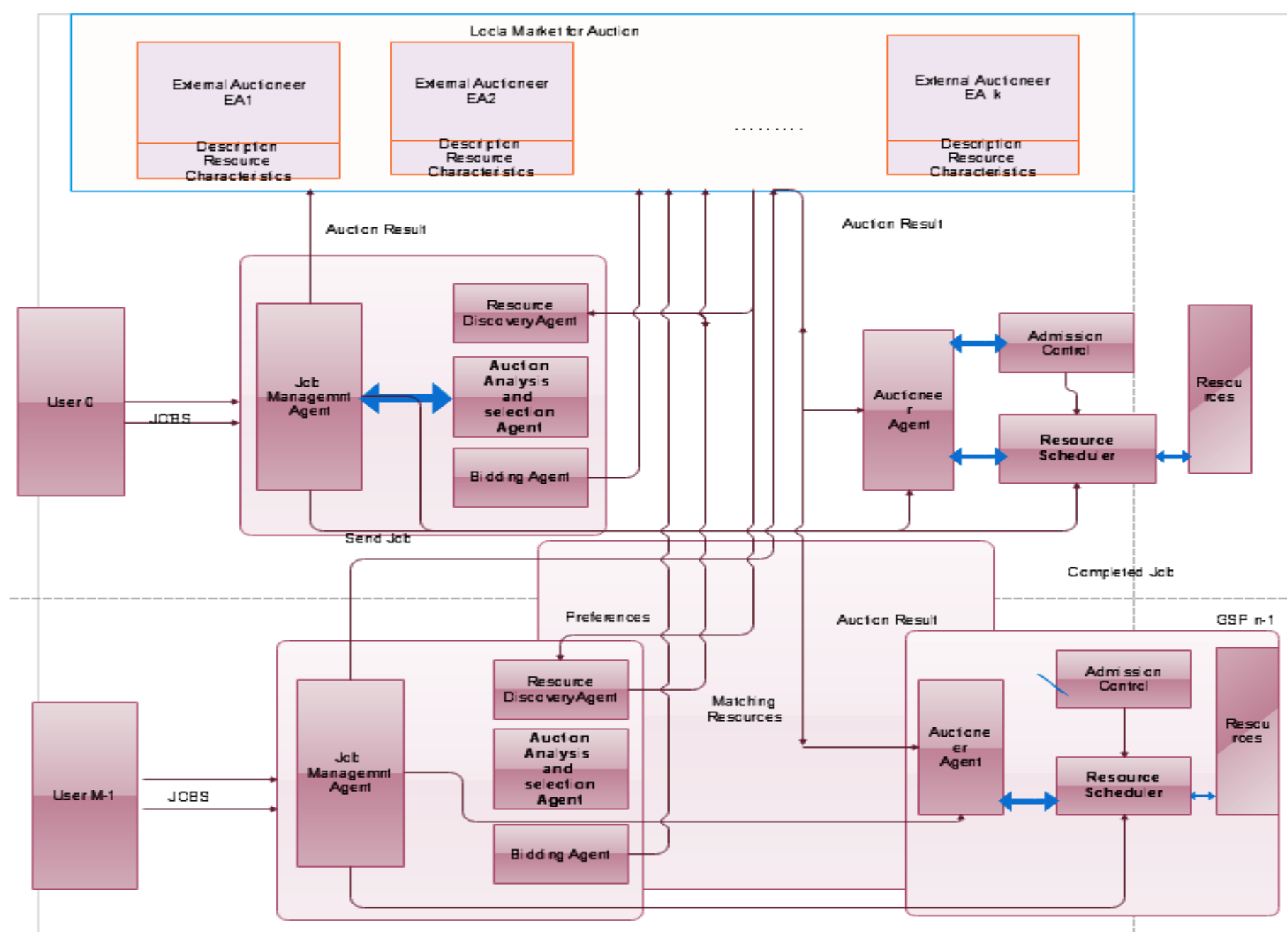

Figure 2: Mergeable auction

the asked for clients, total total to be remunerated via clients, deposit of VM objects allotted to every client in addition to this instrument fundamentally pursues each and every one otherwise no one organization. Moreover arrangement of asked for sources were dispensed otherwise nobody of them is allotted in the direction of clients. Clients could ask in support of their endemic mixes of VMs from beginning to end offers.

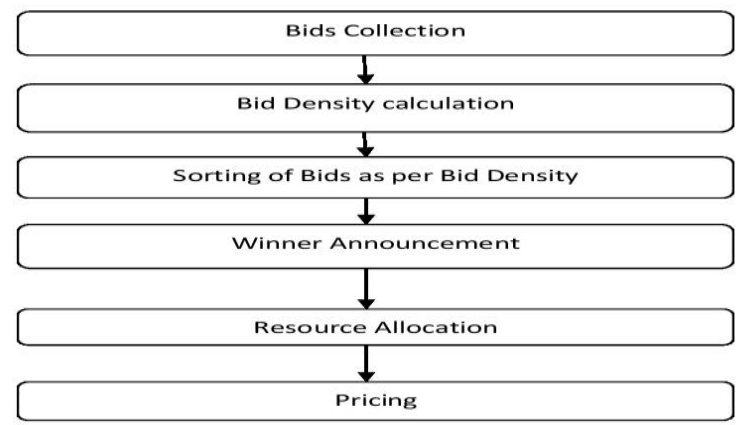

\section{Figure 3: Flow chart in support of MA - PROVISION method}

This method is isolated within triplet noteworthy stages: gathering offers as of clients, champion assurance via thickness computation also compensation. $\mathrm{CC}$ supplier may comprise of $\mathrm{n}$ diverse kinds of VM objects in addition to its heaviness dependent scheduled calculating authority is shown with w. CC suppliers chooses the heaviness in support of sources amid the arrangement of the resources itself. Clients can ready to pick any sort of VM instances they need and offer for the resource into blends like

$$
\mathrm{B}_{\mathrm{j}}=\left(\mathrm{r}_{1, \ldots, \mathrm{rm}}^{\mathrm{j}}, \mathrm{v}_{\mathrm{j}}\right)
$$

This demonstrates the client $\mathrm{j}$ is asking for the offer surrounding $m$ diverse kinds of sources they were inside necessitate. Inside this offer demand, vj is amount client requirements to compensate in support of asked for source.

Champion assurance is completed dependent scheduled count of thicknesses of offers. Thickness of offer is figured via utilizing equation,

$$
\mathrm{D}_{\mathrm{j}}=\mathrm{v}_{\mathrm{j}} / \mathrm{s}_{\mathrm{j}}
$$

Where vj characterizes amount client requirements towards compensate in support of sources in addition to $\mathrm{sj}$ is known through quantity of objects of sources distributed along with heaviness of sources. The offer thickness is too much quantity client requirements towards compensate in support of solitary element of source in necessitate.

The thicknesses of clients were arranged into downward command. Clients among most peak offer were allotted champ in addition to champions were arranged within rundown dependent scheduled their thicknesses. Conditioning of sources is completed based scheduled this arranged request inside 
each and every one otherwise nobody premise. Later than distributing sources in support of a client nature is confirmed in favour of several log jam circumstance. In the event that it is discovered then the rollback task is executed. When every one of sources was given like VM objects towards clients, compensation computation stage begins dependent scheduled use of sources. Clients were always observed also on one occasion their utilization is in excess of they were coordinated in the direction of compensation portal. Expenditure in support of claimed sources whichever is inactive remain additionally considered. Expenditure computation in support of mutually inactive in addition to expended sources remain considered via

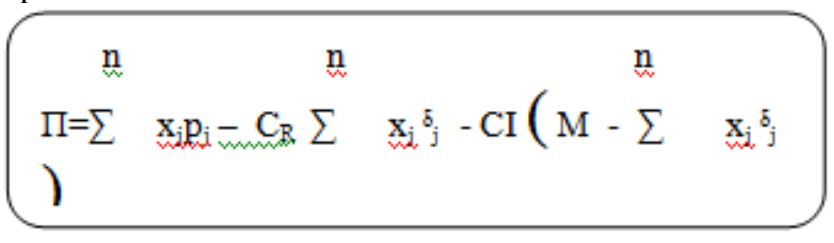

Where CR and CI speaks to the expense of resource at run time and inactive time individually.

As this algorithm pursues dynamic portion, same resource can be assigned to the next client when it isn't being used by the possessed client. This expands the supplier's income in addition to trustworthiness. Aforementioned MA PROVISION method remain honest like it figures sum just dependent onwards the client's utilization as it were.

\section{EXPERIMENTAL RESULTS}

For ease of recreation, we executed mergeable sales by means of triplet assets: RAM, storage, also CPU control. Through the particular triplet assets being mentioned via client, expense on whichever the particular were being given remain appeared beneath separated by a specific moment of instance.

Examined an example recreation of various asset obtainments under successive and mergeable sell-offs. In this recreation, the client details are as per the following: RAM of limits $2 \mathrm{~GB}$ with $4 \mathrm{~GB}$ [8], storage of limits $4 \mathrm{~GB}$, $10 \mathrm{~GB}, 20 \mathrm{~GB}$ and $40 \mathrm{~GB}, \mathrm{CPUs}$ of sort $2 \mathrm{vCPU}, 4 \mathrm{vCPU}$ and $8 \mathrm{vCPU}$. For this solicitation of the client, we executed both successive and mergeable sales. The expense particulars for the acquirement of these assets are yielded Table 1 beneath.

Table 1: Resource Acquirement Expenses

\begin{tabular}{|l|r|}
\hline Resource & Cost \\
\hline 2 GigaByte RandomAccessMemory & $\$ 74$ \\
\hline 4 GigaByte RandomAccessMemory & $\$ 116$ \\
\hline 2 GigaByte HardDiskDrive Cache & $\$ 56$ \\
\hline 10 GigaByte HardDiskDrive Cache & $\$ 66$ \\
\hline 20 GigaByte HardDiskDrive Cache & $\$ 70$ \\
\hline 40 GigaByte HardDiskDrive Cache & $\$ 82$ \\
\hline 80 GigaByte HardDiskDrive Cache & $\$ 100$ \\
\hline 2virtualCentralProcessingUnit & $\$ 212$ \\
\hline 4 virtualCentralProcessingUnit & $\$ 276$ \\
\hline 8 virtualCentralProcessingUnit & $\$ 224$ \\
\hline $\begin{array}{l}\text { 2 GigaByte RAM + 4 GigaByte } \\
\text { HardDiskDrive Cache + 2 } \\
\text { virtualCentralProcessingUnit }\end{array}$ & $\$ 248$ \\
\hline $\begin{array}{l}\text { 4 GigaByte RAM + 5 GigaByte } \\
\text { HardDiskDrive Cache + 4 } \\
\text { virtualCentralProcessingUnit }\end{array}$ & $\$ 358$ \\
\hline $\begin{array}{l}\text { 20 GigaByte HardDiskDrive Cache + } 8 \\
\text { virtualCentralProcessingUnit }\end{array}$ & $\$ 400$ \\
\hline 40 GigaByte HardDiskDrive Cache + & $\$ 160$ \\
\hline
\end{tabular}

80 GigaByte HDD Storage 1

At the same time we executed recreations over the previously revealed client demands via reenacting standards in support of acquirement expenses against quantity of things with it sales, we acquired dual curvatures like appeared Figure 4. In Figure 4, we see that with it curvature for the successive sales, charge for each asset increments consecutively since quantity of assets in the direction of obtained increments. This is essentially since as the quantity of assets to be acquired expands, it includes onto consecutively dependent scheduled greatest cost accessible in support of facilitating asset. It ends up being a solitary asset obtainment issue [8] played out various occasions. Thus the all out expense of acquisition for this demand ends up being $\$ 700$. This demonstrates to be less useful contrasted with asset acquisition performed through a mergeable sale system where various assets that the client demands are secured at a lesser cost than the whole of their person costs.

The curvature for the mergeable sales demonstrates the efficacy of mergeable sales in cloud asset acquisition. As should be obvious during Figure 4, when quantity of assets in the direction of sold expands, mergeable sell off method gives the best cost to the mentioned position of assets. This is supported via way so as towards like numeral of offers CC merchant triumphs increments, additional noteworthy remain quantity of assets so as to remain being auctioned contrasted with so as to of a successive sale, wherever just a single asset is measured on some moment of moment. Thus, this remains exchange wherever equally CC seller in addition to client mentioning in support of asset advantage. Absolute acquisition charge utilizing mergeable sale ends up being $\$ 510$, which is more conservative contrasted with the expense gotten by successive sales. The figuring for this is being lost as uninteresting as it may, unimportant, since it goes through recursive calls as clarified in the calculation.

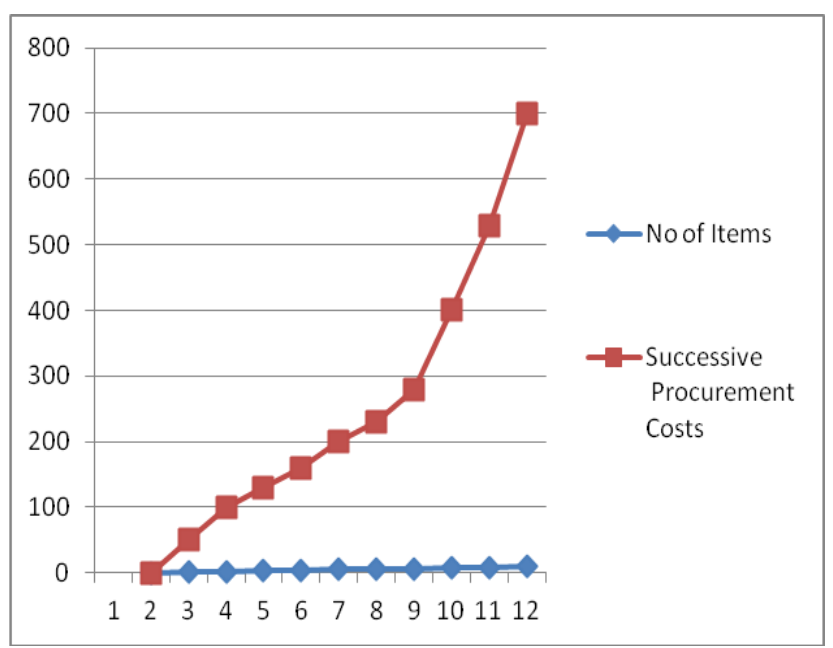

Fig 4. Procurement Costs of successive Sales 


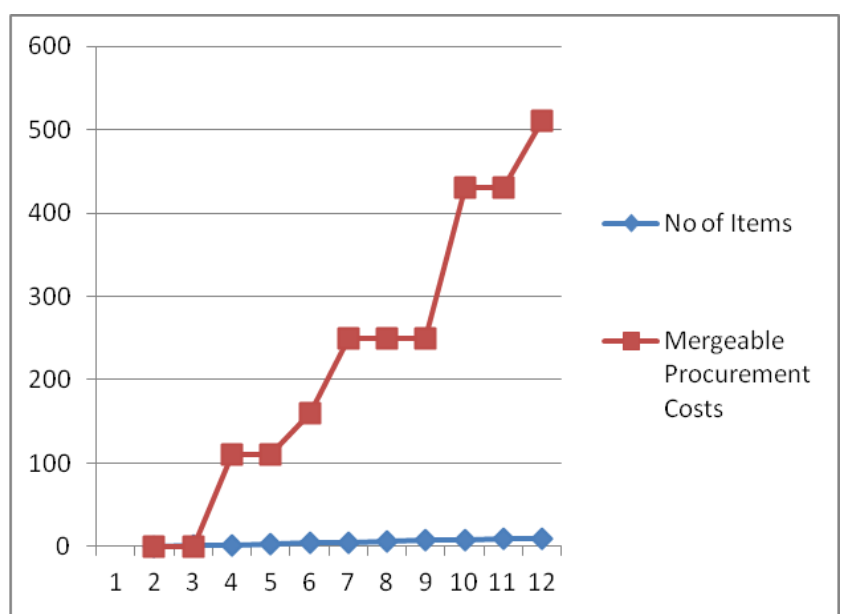

Fig 5. Procurement Costs of Mergeable Sales

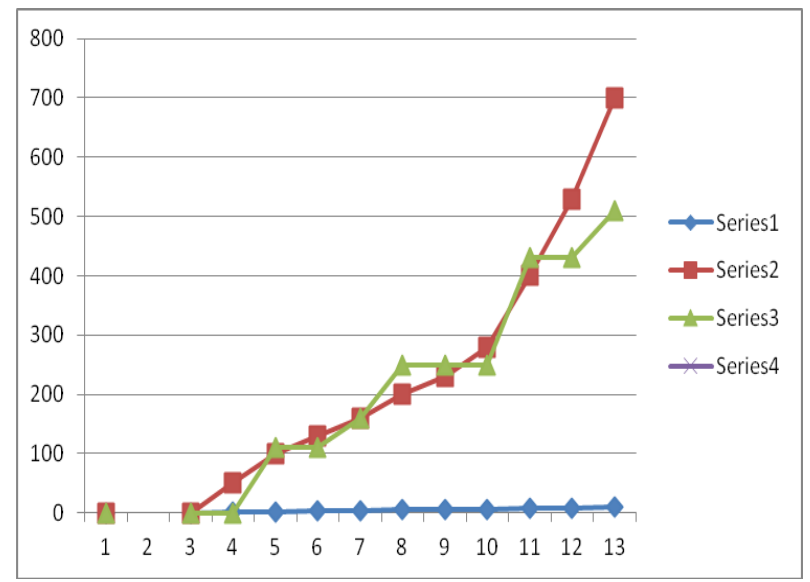

Fig 6. Comparison of Procurement Costs of successive and Mergeable Sales

On looking at the asset acquisition costs among successive sales and Mergeable sell-offs, we can see that as the quantity of assets mentioned by the client increments, Mergeable sell-offs are far better than successive sell-offs, as appeared in Figure 4.

CloudSim tool is utilized for the simulation of cloud condition. It represents demonstrating and reproduction of substantial scale distributed computing server farms and supports re-enactment of virtualized host [8].

The significant utilization of Cloudsim in this project is Cloudsim contains bundles for executing bargainers. In this recreation, five bidders and representatives are taken and the algorithm is connected and results are considered. Results demonstrated that the supplier's income is expanded to certain impressive reach out than the ordinary sales.

Customer's request for movement is likewise diminished because of the blends of resource bundles as opposed to single resource request. Dynamic resource allotment expands the cloud clients and supplier's revocation. Underutilization of resources is limited. Facilitate usage on expanding the quantity of bidders and data centers are under advancement.

\section{CONCLUSION AND FUTURE WORK}

Results showed that this MA - PROVISION algorithm can produce preferred income over alternate algorithms as it depends on unique allotment of resources. Supplier's renouncement likewise expanded because of the honesty of the algorithm. Additionally forms are under execution. Productive valuing components should be considered later on as there may happen some false cases as the cloud condition is principally founded on the web. Resource alongside VM relocation procedures are to be contemplated further. In this paper resource portion by resource is mostly thought. Be that as it may, while actualizing progressively, the significant distributed computing issues like security, protection and heterogeneity ought to be tended to without influencing the execution and accessibility.

\section{REFERENCES}

1. Abinandan S. Prasad and ShrishaRao, "A Mechanism Design Approach to Resource Procurement in Cloud Computing", Proc. IEEE Transactions on Computers, pp. 17-30, 2014.

2. AhuvaMualem and Noam Nisan,"Truthful guess components for confined mergeable auctions,"Proc. eighteenth Nat'l conf. Man-made consciousness, pp. 379-384,2002.

3. Amazon, "Amazon Elastic Compute Cloud (Amazon EC2)," http://aws.amazon.com/ec2/,2013.

4. A. Quiroz, H. Kim, M. Parashar, N. Gnanasambandam, and N. Sharma, "Towards Autonomic Workload Provisioing for Enterprise Grids and Clouds," Proc. IEEE/ACM tenth Int'l Conf. Network Computing, pp. 50-57, 2009.

5. A. Das and D. Grosu, "Mergeable Auction-Based Protocols for Resource Allocation in Grids," Proc. nineteenth Int'l Parallel and Distributed Processing Symp., Sixth Workshop Parallel and Distributed Scientific and Eng. Registering, 2005.

6. D. Lehmann, L. I. Oçallaghan, and Y. Shoham, "Truth Revelation in Approximately Efficient Mergeable Auctions," J. the ACM, vol. 49, no. 5, pp. 577-602, 2002.

7. P. Cramton, Y. Shoham, and R. Steinberg, Mergeable Auctions.MIT Press, 2005.

8. Rodrigo N. Calheiros, Rajiv Ranjan, Anton Beloglazov, Cesar A. F. De Rose and RajkumarBuyya, "CloudSim: A toolbox for demonstrating and recreation of distributed computing conditions and assessment of resource provisioning algorithms," Proc. Wiley Online Library, 2010.

9. S. Zaman and D. Grosu, "Mergeable Auction-Based Allocation of Virtual Machine Instances in Clouds," Proc. IEEE Second Int'l Conf. Cloud Comp. Innovation and Science, pp. 127-134, 2010.

10. S. Zaman and D. Grosu, "A Mergeable Auction-Based Mechanism for Dynamic VM Provisioning and Allocation in Clouds," Proc. IEEE Transactions on Cloud Comp., pp. 129-141, 2013.

11. Sven de Vries and RakeshVohra, "Mergeable Auctions: An overview".

12. YouwenLan, Weiqin Tong, Zongheng Liu, Yan Hou, "Multi-Unit Continuous Double Auction Based Resource Allocation Method," Int'l Conf. Smart Control and Information Processing, pp. 773-777, 2012.

13. V. Vinothina, Dr. R. Sridaran and Dr.PadmavathiGanapathi, "A Survey on Resource Allocation Startegies in Cloud Computing," Proc. Int'l Journal of Advanced Computer Science and Application, pp. 97104, 2012.

14. PeiYun Zhang,Mengchu Zhou,"Dynamic Cloud Task Scheduling Based on a Two-Stage Strategy",IEEE Transactions on Automation Science and Engineering,pp.772-783,2018

15. Mian Guo,Quansheng Guan,Wende Ke,'Optimal Scheduling of VMs in Queueing Cloud Computing Systems with a Hetrogeneous Workload",IEEE Acesss,pp.15178-15191,2018

16. Xiaolong Liu,Shyan-Ming Yuan,Geo-Heng Luo,Hao-Yu Huang,Paolo Bellavista,"Cloud Resource Management with Turnaround Time Driven Auto-Scaling",IEEE Access,pp.98319841,2017 


\section{Effectual Resource Allocation using Auction Mechanism in Cloud Computing}

\section{AUTHORS PROFILE}

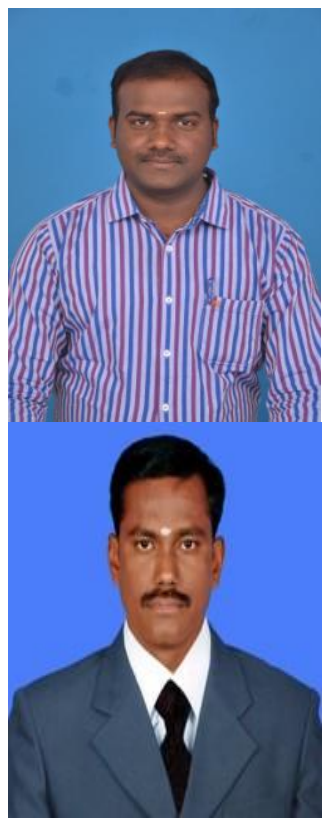

R.Anantha Kumar received his Master of Engineering in 2011 from the Rajalakshmi Engineering College, Anna University, India. $\mathrm{He}$ is currently working as an Assistant Professor in Kalasalingam Institute of Technology, India. His research interests include Cloud Computing, Machine Learning, Big data His publications mainly appeared in international journals and popular conferences

Dr.K.Kartheeban received the Ph.D Degree in 2013 from the Kalasalingam University, India. $\mathrm{He}$ is currently working as an Associate Professor in Kalasalingam Academy of Research Education, India. His research interests include Cloud Computing, Image processing, Big data,Networking. His publications mainly appeared in international journals and popular conferences 